\section{Die indirekte Wirkung von Röntgenstrahlen auf Aminosäuren}

\section{Bestrahlung von Lysin}

Von G. Peter

Max-Planck-Institut für Biophysik, Frankfurt

(Z. Naturforschg. 14 b, 135 [1959] ; eingegangen am 27. Oktober 1958)

Bei der Beurteilung der Grundvorgänge der biologischen Strahlenwirkung tritt die Bedeutung der indirekten Strahlenwirkung immer mehr in den Vordergrund. Im Rahmen der Untersuchungen von sekundären strahlenchemischen Reaktionen bei Aminosäuren und anderen biologisch wichtigen Verbindungen soll in diesem $\mathrm{Be}$ richt über die Ergebnisse der diesbezüglichen, auf Veranlassung von B. Rajewsky durchgeführten Untersuchungen eine kurze Übersicht gegeben werden.

Es wurde die Röntgenstrahlen-Wirkung bei Lysin ( $\alpha, \varepsilon$-Diamino- $n$-capronsäure) untersucht. $\mathrm{Zu}$ diesem Zweck wurden Lysinlösungen verschiedener Konzentration $(0,4 \% ; 0,04 \% ; 18 \%)$ mit der Dermopan-Anlage $(45 \mathrm{kV}, 25 \mathrm{~mA}$ bei einer mittleren Dosisleistung in der wäßrigen Schicht von $7,6 \cdot 10^{4} \mathrm{r} / \mathrm{min}$ bestrahlt) und die bestrahlten Lösungen anschließend mittels Hochspannungs-Elektrophorese ${ }^{1}$ und Papierchromatographie untersucht. Bestrahlt wurde 90 Min., so daß die eingestrahlte Energie $6,8 \cdot 10^{6} \mathrm{r}$ betrug. Außerdem wurde das Lysin mit der Hochleistungs-Röntgenanlage $(80 \mathrm{kV}, 1000 \mathrm{~mA})$ bei einer mittleren Dosisleistung in der wässerigen Schicht von $5,7 \cdot 10^{5} \mathrm{r} / \mathrm{min}$ bestrahlt. Damit war man in der Lage, die gleiche Dosis, nämlich $6,8 \cdot 10^{6} \mathrm{r}$ in 12 Min. zu applizieren.

Bei der 0,4-proz. Lösung zeigte sich, daß neben viel unverändertem Lysin weitere Basen und eine neutrale Fraktion entstanden waren, die mit Ninhydrin positive Reaktionen geben. Diese neuen Komponenten wurden aus den Pherogrammen eluiert und sowohl papierchromatographisch als auch elektrophoretisch mit verschiedenen Aminoverbindungen verglichen. Dabei fand

1 Tн. Wreland u. Mitarb., Angew. Chem. 67, 257 [1955]. man Übereinstimmung der Bestrahlungsprodukte mit folgenden Substanzen:

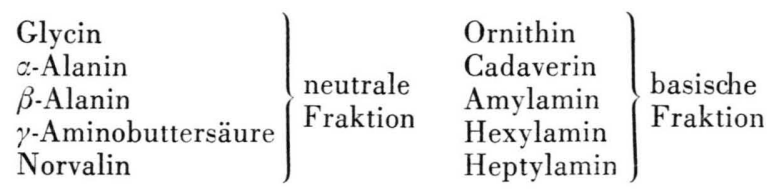

Außer diesen traten noch zwei weitere basische Verbindungen auf, deren Zuordnung bisher nicht mit Sicherheit gelungen ist.

Bei der 0,04-proz. Lösung findet man nicht die Bande der $\gamma$-Aminobuttersäure, als Base nur Ornithin und zusätzlich eine saure Verbindung, die mit der Asparaginsäure identisch sein dürfte.

Nach Bestrahlung einer 18-proz. Lysinlösung findet man ebenfalls keine $\gamma$-Aminobuttersäure, bei den Basen fehlen die Banden des Ornithins und Hexylamins.

Bei diesen strahlenchemischen Umsetzungen handelt es sich mit Sicherheit um die sekundäre Wirkung oxydierender, bei der Wasserspaltung entstandener, Radikale. Dafür spricht auch, daß die beschriebene Umwandlung in eingefrorenen Lysinlösungen nicht eintritt und daß bei Bestrahlung in $\mathrm{O}_{2}$-Atmosphäre die höher oxydierten sauren Spaltprodukte vermehrt auftreten.

Die Radikalwirkung erstreckt sich also auf:

a) Decarboxylierungen,

b) Desaminierungen,

c) Veränderungen des Kohlenstoff-Gerüstes.

Versuche, die Bestrahlungsprodukte eindeutig zu identifizieren, sind im Gang. Die mathematische Behandlung der energetischen Verhältnisse und die theoretischen Betrachtungen zur Deutung der Grundvorgänge sekundärer Strahlenreaktionen werden später publiziert.

Die Untersuchungen wurden im Max-Planck-Institut für Biophysik und im Institut für Organische Chemie der Universität Frankfurt a. M. durchgeführt. Herrn Prof. Dr. B. Rajewskx, Herrn Prof. Dr. Th. Wieland, Herrn Priv.-Doz. Dr. G. Pfleiderer und Herrn Dr. K. Dose danke ich für zahlreiche Diskussionen und Ratschläge.

\section{Über die anaerobe $\mathrm{CO}_{2}$-Bildung wachsender Kulturhefezellen}

\author{
Von Lutz Kiesow
}

Physiol.-chemisches Institut der Freien Universität Berlin (Direktor: Professor Dr. Dr. E. Sснüтте)

(Z. Naturforschg. 14 b, 135-136 [1959]; eingeg. am 5. Dezember 1958)

Beim Übergang von einfacher Salzlösung, wie Phosphatglucose, zu Würze als Milieu zeigen Kulturhefezellen eine beträchtliche Steigerung der anaeroben Gärung und demzufolge auch der aeroben Gärung. Gleichzeitig wird bei diesem Übergang die HexokinaseAktivität der Zellen erhöht ${ }^{1}$.
Da im Verlauf des Zellwachstums in Würze die anaerobe $\mathrm{CO}_{2}$-Bildung noch weiter ansteigt, war zu erwarten, daß auch die Hexokinase-Aktivität proportional erhöht wird. Die Untersuchung der Hexokinase gewachsener Zellen zeigte aber, daß die Aktivität gegenüber dem Wert zu Beginn des Wachstums nicht erhöht, sondern erniedrigt ist.

Dieses, zunächst überraschende Ergebnis versteht man, wenn man von Zellen, die 3 Stdn. in Würze gewachsen waren, die anaerobe $\mathrm{CO}_{2}$-Bildung, die Hexokinase-Aktivität und die gebildeten Mengen an Acetaldehyd und Äthylalkohol mißt. So findet man z. B. für $12 \mathrm{~mm}^{3}$ Bäckerhefezellen in $21 \mathrm{Min}$. bei $20^{\circ} \mathrm{C}$ die Bil-

1 L. Kiesow, im Druck. 
dung von : (Zur Methodik siehe l. c. ${ }^{1}$ ) $9,2 \mu$ Mole $\mathrm{CO}_{2}$.

Die gleichen Zellen bildeten in derselben Zeit:

$4,1 \mu$ Mole Äthylalkohol

und $\quad 0,4 \mu$ Mole Acetaldehyd

$\Sigma$ 4,5 $\mu$ Mole Acetaldehyd + Äthylalkohol.

Aus der gemessenen Hexokinase-Aktivität dieser Zellen errechnet man für $p_{\mathrm{H}} 6,0,20^{\circ}, 12 \mathrm{~mm}^{3}$ Zellen und 21 Min. die Bildung von:

4,4 $\mu$ Mole $\mathrm{CO}_{2}$ oder Acetaldehyd + Alkohol

Über die Hexokinase-Aktivität, die anaerobe
Gärung und das Wachstum von Kulturhefezellen

Von Lutz Kiesow

Physiol.-chemisches Institut der Freien Universität Berlin (Direktor: Professor Dr. Dr. E. Sснüтte)

(Z. Naturforschg. 14 b, 136 [1959] ; eingegangen am 5. Dezember 1958)

Wenn Bäckerhefezellen in Bierwürze wachsen, nimmt das Zellvolumen bei hinreichend kleinen Aussaaten exponentiell von der Zeit 0 an ${ }^{1}$ bis zu einem bestimmten Endwert zu. Bei diesem Endzustand findet man fast keine weitere Volumenzunahme der Zellen und für ihn wird die sog. „Eigenhemmung“ des Wachstums verantwortlich gemacht ${ }^{2}$.

Man kann diesen Wachstumstillstand auch dadurch erreichen, daß man Würze mit einer relativ großen Zellmenge kurze Zeit inkubiert, die Zellen abzentrifugiert und nun eine kleine Zellmenge in diese Würze erneut aussät, oder daß man die Würze mit einem Kationenaustauscher vor der Aussaat einer kleinen Hefemenge behandelt. Ein Vergleich der anaeroben $\mathrm{CO}_{2}$-Bildung und der Hexokinase-Aktivität von Zellen, die in der so vorbehandelten Würze nicht wachsen, mit

1 L. Kiesow, im Druck. über den Embden-M e y e r h of - Weg der Glykolyse. Danach ist die Hexokinase-Aktivität der gewachsenen Kulturhefezellen nur noch dem anaerob gebildeten Acetaldehyd + Alkohol proportional und nicht mehr dem anaerob gebildeten Kohlendioxyd. Es müssen also:

$$
9,2-4,5=4,7 \mu \text { Mole } \mathrm{CO}_{2}
$$

anders als aus der Decarboxylierung der Brenztraubensäure entstanden sein. Die Hexokinase aber bestimmt auch bei diesen Zellen die Größe der Gärung.

Die Versuche werden an anderer Stelle ausführlich dargestellt.

den gleichen Größen von Hefezellen, die in PhosphatGlucose-Lösung suspendiert sind, zeigt folgendes:

1. In Würze (durch Inkubation mit lebenden Hefezellen vorbehandelt) :

$$
W_{\text {Hexokinase }}=7,2 \cdot 10^{-3} \cdot \mathrm{min}^{-1} \cdot \mathrm{mm}^{-3} Q_{\mathrm{CO}_{\mathrm{g}}}^{\mathrm{Ar}}=107 .
$$

2. In Würze (mit Kationenaustauscher vorbehandelt): $W_{\text {Hexokinase }}=5,8 \cdot 10^{-3} \cdot \mathrm{min}^{-1} \cdot \mathrm{mm}^{-3} Q_{\mathrm{CO}_{2}}^{\mathrm{Ar}}=92$.

3. In Phosphat-Glucose-Lösung:

$$
W_{\text {Hexokinase }}=6,7 \cdot 10^{-3} \cdot \mathrm{min}^{-1} \cdot \mathrm{mm}^{-3} Q_{\mathrm{CO}_{2}}^{\mathrm{Ar}}=101 .
$$

Sowohl die Hexokinase-Aktivität als auch die anaerobe Gärung stimmen annähernd bei allen Medien überein. Die für die Würze charakteristisch gefundene Erhöhung der anaeroben Gärung und der HexokinaseAktivität der Zellen gegenüber Phosphat-Glucose-Lösung ist durch die Vorbehandlung der Würze verschwunden und die Zellen wachsen nicht mehr.

Man wird deshalb aus diesen Versuchen folgern, $\mathrm{da} \beta$ in dem einen Fall die lebenden Hefezellen und in dem anderen der Ionenaustauscher ein Prinzip der Würze gebunden haben, das die Hexokinase-Aktivität und damit die Gärung erhöht und das Wachstum auslöst.

2 W. Scнмid, Z. Naturforschg. 7 b, 217 [1952].

\section{Färbung mit TTC (Triphenyltetrazoliumchlorid) an der Larve von Ephemera unter aeroben Bedingungen bei $28^{\circ} \mathrm{C}$}

\section{Von Otтo Harnisch}

Hydrobiologische Anstalt der MPG zu Plön

(Z. Naturforschg. 14 b, 136-137 [1959] ; eingeg. am 24. November 1958)

Im Sommer und Herbst 1958 hat Fräulein Ковг (Lubljana), die an unserem Institut arbeitete und ihre Ergebnisse später veröffentlichen wird, in Zusammenarbeit mit mir einige Beobachtungen gemacht, deren Verfolgung und Veröffentlichung sie mir freundlichst überließ. Jedenfalls habe ich ihr für ihre sorgsame Mitarbeit auf herzlichste zu danken. - Ich bin mir darüber im klaren, daß die im folgenden geschilderten Beobach- tungen nicht allgemein für jede Ephemera-Larve Gültigkeit haben, sondern an einigen glücklichen, vielleicht aber nicht seltenen Fällen gemacht werden.

Bei den Studien an der Ephemera-Larve zeigte sich, $\mathrm{da} ß$ die Larven bei erhöhter Temperatur $\left(28^{\circ} \mathrm{C}\right)$ schon beim Partialdruck der Luft gelegentlich zur Abgabe eines durch $\mathrm{KOH}$ nicht absorbierbaren Gases (wohl $\mathrm{O}_{2}$ ) neigen. Ich habe in verschiedenen Mitteilungen ${ }^{1}$ die Vermutung geäußert, daß an den Einschlußkörpern des Fettkörpers von Insekten - namentlich ChironomidenLarven in Zusammenhang mit der Gasabgabe Reduktionsprozesse ablaufen, die eine Rotfärbung von TTC zur Folge haben. Dies wurde an euroxybionten Chironomiden-Larven unter anaeroben, kaum aber unter

\footnotetext{
1 Vgl. u. a. Zool. Jb. Physiol., 66, 547 [1956].
} 